\title{
Palatability Testing of Oral Chewables in Veterinary Medicine for Dogs
}

\author{
Michelle Aleo' ${ }^{1}$, Stacy Ross ${ }^{2}$, Csilla Becskei ${ }^{3}$, Eileen Coscarelli1, Vickie King ${ }^{4}$, Mary Darling1, \\ Julie Lorenz ${ }^{2}$
}

${ }^{1}$ VMRD Global Therapeutics, Zoetis, Kalamazoo, MI, USA

${ }^{2}$ VMRD Lab Sciences, Zoetis, Kalamazoo, MI, USA

${ }^{3}$ VMRD Global Therapeutics, Zoetis, Zaventem, Belgium

${ }^{4}$ VMRD Global Development and Operations, Zoetis, Kalamazoo, MI, USA

Email: michelle.m.aleo@zoetis.com, mary.l.darling@zoetis.com, julie.k.lorenz@zoetis.com, csilla.becskei@zoetis.com,

vickie.1.king@zoetis.com

How to cite this paper: Aleo, M., Ross, S., Becskei, C., Coscarelli, E., King, V., Darling, M. and Lorenz, J. (2018) Palatability Testing of Oral Chewables in Veterinary Medicine for Dogs. Open Journal of Veterinary Medicine, 8, 107-118.

https://doi.org/10.4236/ojvm.2018.88011

Received: May 5, 2018

Accepted: August 25, 2018

Published: August 28, 2018

Copyright $\odot 2018$ by authors and Scientific Research Publishing Inc. This work is licensed under the Creative Commons Attribution International License (CC BY 4.0).

http://creativecommons.org/licenses/by/4.0/

\begin{abstract}
For veterinary medications administered per os, animal health companies strive to develop highly palatable dosage forms that are voluntarily accepted by animals to improve compliance and convenience. Achieving high palatability is often complex and difficult even without the presence of an active ingredient. This work compared acceptance and preference studies, as standardized methods are not established for informing formulation development or for more routine testing. Formulation development was followed by an acceptance study completed with laboratory Beagle dogs. One acceptance study and one preference study were completed in mixed breed dogs, also laboratory-housed, to gain wider representation of dog breed and age. Through these studies, we have evaluated both formulation parameters and palatability study conduct. In general, more complex palatants that have appealing taste, smell, and mouth feel enhance voluntary uptake. However, dosage forms that are too chewy may not be freely consumed even with complex palatants. The addition of aroma can entice dogs to prehend the tablet, as observed in one preference study. Preference studies in the veterinary pharmaceutical field identify the preferred first choice or first prehend, but not which product will be routinely voluntarily and fully consumed. Acceptance studies with cross-over treatment groups are used to quantify the full consumption of a dosage form when a dog is not given two choices at once. Since all dogs in acceptance studies are offered all treatment groups throughout the study, a comparison between degrees of consumption could suggest that one formulation might be preferred over another.
\end{abstract}




\section{Keywords}

Palatability, Acceptance, Preference, Dog, Veterinary

\section{Introduction}

For veterinary oral dosage forms, compliance and convenience can be challenges that are critical for prevention, control, and treatment medication programs. This is especially true for tablets or capsules given to companion animals that are administered via the traditional "poke down" method or hidden in an attractive food, such as cheese or peanut butter. Ideally, the dosage form would be voluntarily consumed from the pet owner's hand or the pet's bowl. These products can be given in different regimens, such as once monthly or daily; and for variable durations, some even continually or chronically. For this reason, veterinary pharmaceutical companies strive to develop products with high palatability.

"Palatable" is defined as the property of being acceptable to the mouth, "pleasant to the taste" or "acceptable to the taste". When applied to a veterinary medicinal product, this term suggests that the product is palatable enough to ensure voluntary uptake [1]. A palatable dosage form can require complex palatants, such as a cocktail of animal-origin proteins, vegetable proteins, sugar, etc. Palatants and excipients in the dosage form can affect palatability. Palatability involves taste, smell, and mouth feel such as: texture, size, or chewiness to entice the dog to consume it. Voluntary acceptance is defined as the willingness of the target animal to voluntarily and spontaneously consume the veterinary medicinal product from a bowl, the ground or from a hand when offered as a treat by the animal owner [1]. For a palatability label claim according to the Committee for Medicinal Products for Veterinary Use (CVMP) Guidance, the product must be voluntarily consumed by at least $80 \%$ of dogs or at least $70 \%$ of other animals within 2 minutes [1]. For veterinary registration palatability studies, compliance is directly measured through acceptance of the dosage form from the bowl or person's hand. While registration palatability studies have guidance, there is not a standard method for studying palatability when developing a palatable dosage form.

The pet food, pet treat, and veterinary medicine fields continue to investigate how to measure consumption, first choice, preference, and behaviors that would indicate excitement. In all of these fields, there are two main types of palatability studies: acceptance (choice to consume or not consume one dosage form) and preference (choice between two dosage forms). An acceptance study consists of a single dosage form being offered per animal, which can be run as a single treatment test or can be run as a cross-over study with multiple treatment groups such that all animals in the study are ultimately offered each treatment. For example, with a two-treatment study of a daily treatment dosage form, half of the animals would receive the first treatment and the other half would receive the 
second treatment during the first period. During the next period, the treatment groups would be switched. Because there is no standardized procedure to test acceptance, different studies use different methods. In some studies the test materials are offered from the animal's bowl, while in other studies they are offered from a person's hand. The studies also vary by the time allowed for the animal to consume the product (60 seconds to 30 minutes) [2] [3]. In general it is evaluated whether an animal fully consumes the offered dosage, does not consume it at all, or only consumes part of it within the maximum time allowed. The advantage to the acceptance test is that it is a direct measure of pet compliance for consuming the medication [4].

A preference test is run with two dosage forms being offered simultaneously to each animal. The aim of the study is to determine the relative palatability of the tested products, and should not be confused with the voluntary acceptance results generated from a "one bowl" acceptance test. In a preference test, the animal is allowed to make a choice between two products. These two products could both have excellent palatability, could have low palatability, or could be different in palatability. In testing of veterinary pharmaceutical products, once a choice is made and one of the products is taken into the mouth, the other product that was not chosen is removed as it may be unsafe to allow the animal to consume both products [3] [5]. The relative preference is calculated in these studies; this preference is known as first approach or first choice in the pet food and treats industry [6]. Since the pet food industry allows both choices to be consumed, the first choice can be differentiated from the overall preferred product through comparing consumption ratios. The pet food industry also uses preference studies to determine a consumption ratio, which is not feasible with medicinal products as it is unsafe to allow the dogs to consume both treatments in unlimited amounts.

The current studies focus on achieving high palatability in formulation development of oral dosage forms rather than comparing marketed products. These studies were performed to understand the challenge of achieving palatable veterinary tablets (even those without active ingredients). Data in this publication suggest that smell is the main element for first choice and demonstrates the learnings from an acceptance study and a preference study using the same treatment groups.

\section{Methods}

\subsection{Formulation Finding and Study 1}

The palatability studies were approved prior to study start by the Zoetis Kalamazoo Institutional Animal Care and Use Committee.

Purpose-bred Beagle dogs were used to screen compounds for acceptance. This breed was readily available for use on-site, and is commonly bred for research purposes due to size and temperament. The authors find this colony of dogs to be sufficient for screening purposes prior to testing in a larger, more va- 
riable population of dogs. Dogs were approximately 1 to 6 years of age and body weights ranged from 6.5 to $13 \mathrm{~kg}$. Neutered males and spayed and intact females were included on-study. Dogs were housed individually during morning feeding and study offerings, but were otherwise placed in pairs or were group-housed to encourage play and socialization. The time of day for the offering, the time of meal feeding, and husbandry activities were conducted at approximately the same time each day.

A total of 16 - 24 dogs were used for each acceptance study. The dogs were offered the investigational products, which contained conventional tablet excipients without active ingredients, for one to three consecutive days. A commercial product or treat of known and predictable acceptance was offered prior to and following any testing period.

Each animal was offered a commercial product or investigational product using identical procedures. The marketed or investigational products were removed from packaging and placed in a clean bowl which was immediately offered to each dog by an individual the dog was familiar with. All treatment offerings were handled with forceps to avoid contamination of taste or aroma. The consumption observation category was then recorded as full, partial, or no. If the test article was still present as partial or no after a two minute time limit, then all material was removed from the dog's bowl and discarded. The percentage of animals in each consumption observation category was calculated for each treatment in each of the studies.

Before, between, and after any treatment periods, a commercial product or other formulation of known acceptance was offered using the same administration procedure and consumption recording as listed above. These data were summarized and classified as additional information.

\subsubsection{Formulation Finding: Studies A-D}

Examples of preliminary learnings on formulations can be summarized in Studies A-D as shown below in Table 1. Each of these individual studies measured voluntary acceptability of a placebo using complex palatants from an identical lot. These results were obtained either from a study with only one treatment group, a study with multiple treatment groups executed with a cross-over design, or between periods in various crossover studies. In all cases, all animals within a study were offered each of the treatment(s) tested.

Table 1 includes two different placebos to help in understanding that palatability consists of both taste and smell. All of the placebos contain traditional pharmaceutical tableting excipients. The treatment group of the tablet with a simple sugar palatant focused on taste. This was compared to a placebo that was used consistently across studies. This second placebo type contained a more complex mixture of palatants for taste and smell.

\subsubsection{Study 1 Acceptance Study}

Study 1 examines three chewable placebos with the goal of assessing the acceptability of palatants of animal and non-animal origin. Twenty-four (24) dogs were 
Table 1. Summary of studies which include a single lot of a placebo with complex palatants to examine the effects of smell and taste on voluntary acceptance.

\begin{tabular}{|c|c|c|c|c|}
\hline & $\begin{array}{c}\text { Number } \\
\text { of dogs }\end{array}$ & Offering regimen & $\begin{array}{c}\text { Total \# of } \\
\text { offerings per dog }\end{array}$ & $\begin{array}{l}\text { Time allowed for } \\
\text { consumption }\end{array}$ \\
\hline $\begin{array}{c}\text { Study A } \\
\text { (Sugar placebo) }\end{array}$ & 16 & $\begin{array}{c}\text { Offered as a } \\
\text { single-treatment study }\end{array}$ & 3 & $2 \mathrm{~min}$ \\
\hline $\begin{array}{c}\text { Study A } \\
\text { (Complex palatant placebo) }\end{array}$ & 16 & $\begin{array}{l}\text { Offered immediately prior } \\
\text { to the study start }\end{array}$ & 1 & $2 \mathrm{~min}$ \\
\hline Study B & 24 & $\begin{array}{c}\text { Offered as } 1 \text { of } 3 \\
\text { treatments in a cross-over } \\
\text { study design }\end{array}$ & 1 & $2 \min$ \\
\hline Study C & 24 & $\begin{array}{l}\text { Offered between cross-over } \\
\text { periods }\end{array}$ & 1 & $2 \mathrm{~min}$ \\
\hline Study D & 24 & $\begin{array}{c}\text { Offered as } 1 \text { of } 2 \\
\text { treatments in a cross-over } \\
\text { design }\end{array}$ & 1 & $2 \min$ \\
\hline
\end{tabular}

offered one of three treatments using a crossover design such that all dogs were offered each treatment over the course of the study. Each dog was assigned to one of six sequences to determine the order of treatment offerings. Treatments were offered daily for three consecutive days in each period.

Two of the treatments contained complex palatants for taste and smell. One of these contained a palatant of animal origin, and the other contained a palatant of non-animal origin. The third treatment was a control placebo $(\mathrm{CP})$ that contained the same complex palatants, but did not have additional excipients that were attributed to the chewy nature of the dosage form and none were added to aid taste or smell.

\subsection{Studies 2 and 3}

Ethical review of the studies occurred prior to in vivo work, and in accordance with all relevant national and international animal welfare regulations. The studies were approved by the Zoetis Ethical Review Team.

These studies used mixed-breed/pure bred dogs that were housed in individual pens. The goal of these two studies was to examine the impact of aroma in sugar placebo tablets on acceptance and first-choice preference. On each day the test procedure for each dog commenced at approximately the same time $( \pm 2$ hours) and at least 4 - 5 hours after the main meal of the dog. At all times during preparation for the tests, contact between the tablet and human hand(s) was avoided; during preparation of the bowl, the tablets were handled by forceps. After each dog had completed the acceptance test, the bowl was thoroughly wiped with a piece of kitchen paper and/or washed with plain water as required. Each aroma placebo tablet contained the same total amount of aroma by weight, and contained traditional tablet excipients.

\subsubsection{Study 2 Acceptance Study}

In total, 48 dogs of various breeds and mixed breeds were selected for the study: 
eleven Beagles, one Border Collie, three Spaniels, one Dachshund, two Fox Terriers, one Pomeranian, one Miniature Schnauzer, two Jack Russell Terriers and twenty-six various unspecified mixed breeds. The age of the dogs ranged between 1 and 18 years, and their weights ranged between 7.0 and $29.7 \mathrm{~kg}$. Intact and neutered males and spayed and intact females were included in the study.

The study was conducted to investigate whether the addition of an aroma component enhances the acceptance of a chewable placebo. The study used a three period cross-over design. Animals were randomized to one of six possible sequences in the three period crossover study for treatments T01, T02, and T03 (see Table 2). The sequences assigned the order of treatments that an animal received, with one treatment per period. All animals received treatment CP (Control Placebo) between periods. A single tablet of a chewable tablet formulation containing one of three different aroma components (T01: Aroma 1, T02: Aroma 2, or T03: Aroma 3) was offered in three series of acceptance tests. Aroma 3 consisted of a combination of Aroma 2 plus a different aroma. Tests were conducted once a day over three consecutive days, with a four day washout period between the series. Each animal received one of the three flavored placebo tablets (T01, T02, or T03) throughout the same test period of three days. The day when the animals first received an aroma placebo tablet was defined as Day 0. The sequence in which the three products were offered on the four days was allocated to dogs randomly. All dogs received a Control Placebo (CP) formulation that did not contain any aroma component on six study days (Days-2, -1, and during the last two days of the washout periods on days $5,6,12$, and 13).

The order in which individual dogs were tested was the same on each occasion. On each day the test procedure for each dog commenced at approximately the same time ( \pm 2 hours) and at least 4 - 5 hours after the main meal of the dog. In the acceptance tests a single tablet of the test material was offered from a bowl to each dog. If the product was not taken into the mouth within 60 seconds of the beginning of the procedure, the procedure was terminated. The prehension of the test material (Yes or No), and whether the dog consumed the tablet (fully, partially, or not at all) was recorded. The percentages of prehension and tablet consumption were calculated for each treatment.

Table 2. Acceptance palatability study results comparing a placebo with sugar for taste and a placebo with complex palatants for taste and smell.

\begin{tabular}{cccc}
\hline & \multicolumn{3}{c}{ Percent of Dogs per Observed Category } \\
\cline { 2 - 4 } Treatment & \multicolumn{3}{c}{ Category } \\
\cline { 2 - 4 } & Full & Partial & None \\
\hline Study A: Placebo-Sugar & 45.8 & 37.5 & 16.7 \\
Study A: Placebo-Complex Palatants & 93.8 & 0.0 & 6.3 \\
Study B: Placebo-Complex Palatants & 95.9 & 4.2 & 0.0 \\
Study C: Placebo-Complex Palatants & 95.8 & 2.1 & 2.1 \\
Study D: Placebo-Complex Palatants & 95.9 & 0.0 & 4.2 \\
\hline
\end{tabular}




\subsubsection{Study 3 Preference Study}

In total, 48 dogs of various breeds and mixed breeds were selected for the study during a two day training session in which two different non-pharmaceutical products were offered to each dog simultaneously. Breeds included: thirteen Beagles, one Belgian Shepherd, four Spaniels, one Fox Terrier, one Shetland Sheepdog, one Miniature Schnauzer, two Welsh Corgis, one Labrador Retriever and 24 various unspecified mixed breeds. The age of the dogs ranged between 2 and 17.8 years, and their weights ranged between 7.2 and $30.9 \mathrm{~kg}$. Intact and neutered males and spayed and intact females were included in the study.

This study examined the impact of aroma on first-choice preference. The study consisted of four days. Animals were randomized to 1 of 24 possible sequences in a four period crossover study. The sequences assigned the order of the treatments that an animal received in each period. Half of the animals receiving the same treatments on a given day were randomized to receive the treatment in the right bowl, with the other half receiving that same treatment in the left bowl. All animals received the placebo in the opposite bowl from the treatment. All dogs were offered one single tablet of the Aroma Placebos (T01: Aroma 1, T02: Aroma 2, T03: Aroma 3, or T04: Aroma 4) with the single Control Placebo (CP) tablet simultaneously on any one day. All aroma placebos were tested once against the $\mathrm{CP}$. The sequence in which the four aroma placebos (T01, T02, T03, and T04) were offered on the four days was randomly allocated to the dogs. The two products that were offered simultaneously on any one day were randomly allocated to the left or right bowl of the test tray. After the test tray was positioned inside the animal's pen, the dog was allowed one minute to choose one of the two products offered to consume. As soon as the dog prehended one product, the test tray was removed from the pen immediately.

Dogs that made a choice in any of the preference tests (taking one product into their mouth) were included in the data summaries and were defined as prehending the product. The number and percentage of dogs preferring each product were calculated for T01, T02, T03, and T04 separately from the control placebo as follows in Figure 1.

\section{Results}

\subsection{Formulation Finding: Studies A-D}

The sugar placebo was fully consumed by $45.8 \%$ of the dogs when offered one time to all dogs prior to the start of Study A. This was approximately half the acceptance rate of the more complex tablet (45.8\% compared to $93.8 \%$ - 95.9\%). The sugar tablet had a higher percentage of dogs that would not voluntarily consume any of the dose $(16.7 \%$ compared to $0.0 \%-6.3 \%)$. More notable was the observation that more dogs would only partially consume the sugar placebo (37.5\% compared to $0.0 \%-4.2 \%)$, rather than fully consume it.

\subsection{Study 1}

Table 3 summarizes the acceptance results of the three placebo tablets tested. 
$\frac{\text { (Number of dogs first prehending } \mathrm{T} 01 / \mathrm{T} 02 / \mathrm{T} 03 / \mathrm{T} 04 \text { or } \mathrm{CP} \text { ) }}{\text { Number of all dogs that chose to prehend a product }} \times 100$

Figure 1. Calculation equation used to determine percentage of acceptance among the dogs which chose to prehend a product.

Table 3. Acceptance palatability study results comparing placebos with complex palatants for taste, smell, and mouth feel (chewiness).

\begin{tabular}{cccc}
\hline & \multicolumn{3}{c}{ Percent of Dogs per Observed Category } \\
\cline { 2 - 4 } Treatment & Full & Partial & None \\
\cline { 2 - 4 } & 94.4 & 4.2 & 1.4 \\
\hline $\begin{array}{c}\text { Control Placebo-Complex Palatants; } \\
\text { Not too Chewy }\end{array}$ & 73.3 & 15.5 & 11.3 \\
$\begin{array}{c}\text { Placebo-Complex Palatants of Animal } \\
\text { Origin; Chewy }\end{array}$ & 22.6 & 64.8 & 12.7 \\
\hline $\begin{array}{c}\text { Placebo-Complex Palatants of } \\
\text { Non-Animal Origin; Chewy }\end{array}$ & & & \\
\hline
\end{tabular}

*Treatments were tested in 24 dogs on three occasions $(n=72)$.

The chewable with non-animal origin had a much lower acceptance rate (22.6\%) than both the control placebo and the placebo with animal-origin palatants added ( $94.4 \%$ and $73.3 \%$, respectively). While the control placebo had high acceptance and was observed to be immediately and effortlessly consumed, this was not the case with the other two placebos formulated with additional excipients. They were determined to be too chewy for the dogs and many were visibly attempting to chew the dosage over the allowable time to consume and continued to show interest in the product. When compared to the control placebo, more offerings of the chewy placebos were only partially consumed within the time allowed (15.5\% of animal-origin chewable and $64.8 \%$ of non-animal origin placebo), or were not consumed at all (11.3\% of animal-origin chewables and $12.7 \%$ of non-animal origin chewables).

\subsection{Study 2}

Table 4 and Table 5 depict the aroma prehension and acceptance study data for a tablet with sugar for taste and those tablets which included at least one aroma ingredient. The addition of the aromatic ingredient did not greatly increase the prehension (75.2\% - 75.9\% compared to control at $69.9 \%)$ or the full consumption $(66.7 \%-68.3 \%$ compared to control at $61.4 \%)$.

\subsection{Study 3}

Table 6 summarizes the results of the aroma preference (first choice) study data and shows which product was prehended first and the first choice frequency. In the 48 preference tests, 23 (47.9\%), 24 (50.0\%), 23 (47.9\%) and 26 (54.2\%) dogs did not take any product to their mouth, when T01, T02, T03 and T04 was offered (respectively simultaneously with the Control Placebo tablet). In the tests 
Table 4. Prehended sugar placebos with and without aromas added.

\begin{tabular}{ccccc}
\hline & \multicolumn{5}{c}{ Percent of Dogs per Observed Category } \\
\cline { 2 - 5 } Treatment & \multicolumn{4}{c}{ Prehended } \\
\cline { 2 - 5 } & \multicolumn{3}{c}{ NO } & \multicolumn{3}{c}{ YES } \\
\cline { 2 - 5 } & $\boldsymbol{n}$ & \% & n & \% \\
\hline CP: Placebo-Sugar & 86 & 30.1 & 200 & 69.9 \\
T01: Aroma 1 & 34 & 24.1 & 107 & 75.9 \\
T02: Aroma 2 & 35 & 24.8 & 106 & 75.2 \\
T03: Aroma 3 & 35 & 24.6 & 107 & 75.4 \\
\hline
\end{tabular}

Table 5. Acceptance palatability study results for sugar placebo tablets with or without (control) the addition of aroma for smell.

\begin{tabular}{cccc}
\hline & \multicolumn{3}{c}{ Percent of Dogs per Observed Category } \\
\cline { 2 - 4 } Treatment & Full & Category \\
\cline { 2 - 4 } & 61.4 & Partial & None \\
\hline CP: Placebo-Sugar & 68.1 & 1.8 & 36.8 \\
T01: Aroma 1 & 66.7 & 0.7 & 31.2 \\
T02: Aroma 2 & 68.3 & 4.3 & 29.1 \\
T03: Aroma 3 & 3.5 & 28.2 \\
\hline
\end{tabular}

${ }^{*}$ CP was tested in 48 dogs on six occasions $(\mathrm{n}=286)$; T01 was tested in $46-48$ dogs on three occasions $(\mathrm{n}=$ 141); T02 was tested in 47 dogs on three occasions $(n=141)$; T03 was tested in $47-48$ dogs on three occasions $(\mathrm{n}=142)$.

where a choice was made, $72.0 \%$ of the dogs preferred T01 (Aroma 1), $66.7 \%$ preferred T02 (Aroma 2) and 63.6\% of the dogs preferred T04 (Aroma 4) over the Control Placebo, while $60.0 \%$ preferred the CP over T03 (Aroma 3). In general, these tablets with aroma where chosen first twice as often as the control. Interestingly, the control was prehended first by $60 \%$ of the dogs compared to the tablet with Aroma 3.

\section{Discussion}

The CVMP guideline establishes parameters for palatability study design in order to be predictive of a field population. In our experience, formulation finding studies are an important step in understanding how palatants and other excipients affect product acceptability. Study protocols can be tailored to help determine whether to rule out, modify, or select formulations for further testing. These data can be compared to a formulation of known acceptance. The complex palatant tested in Studies A-D consistently outperformed a sugar placebo known to be accepted at less than $50 \%$ by in-house laboratory Beagles. While results are not generally compared across studies, consistency in the acceptance of the same complex placebo tablet across time and study design was an important finding in building confidence in the data. With these study designs, the 
Table 6. Preference palatability study results for sugar placebo tablets compared to tablets with aroma for smell.

\begin{tabular}{cccc}
\hline \multirow{2}{*}{ Treatment $^{*}$} & \multicolumn{3}{c}{ First Treatment Prehended } \\
\cline { 2 - 4 } & Sugar Placebo Control & Aroma Tablet & $\begin{array}{c}\text { First Choice } \\
\text { Frequency }\end{array}$ \\
\hline T01: Aroma 1 & 28.0 & 72.0 & 2.6 \\
T02: Aroma 2 & 33.3 & 66.7 & 2.0 \\
T03: Aroma 3 & 60.0 & 40.0 & 1.5 \\
T04: Aroma 4 & 36.4 & 63.6 & 1.7 \\
\hline
\end{tabular}

*The number of tests was 48 for each test product with 24 dogs.

most palatable formulation can be determined and used for further studies.

For a palatable pharmaceutical oral dosage form (tablet, hard chew, soft chew, gel, etc.), the most critical test is the acceptance test. It is a direct measure of compliance of the dog voluntarily consuming the medication. In the formulation finding studies, the traditional pharmaceutical tablet excipients with the addition of sugar were voluntarily consumed in less than half of the offerings. Thus, a veterinary sugar placebo dosage form (without drug) is assumed to not have a high palatability. Including more complex palatants to the dosage form for taste and smell approximately doubled the acceptance of the tablet as shown in Studies A-D. This was confirmed with the in-house acceptance palatability study (Study 1) that used a different tablet formulation with similar complex palatants and led to voluntary consumption in $94.4 \%$ of the offerings. In addition to taste and smell critically influencing palatability, the mouth feel can also play a significant role. In the same study, even in the presence of complex palatants for taste and smell, the treatments were not well accepted when the dosage form was too chewy.

The sugar placebo tablet used for comparison in the formulation finding studies was also tested in a mixed breed dog colony (Studies 2 and 3). The Beagle colony is exclusively used to test pharmaceutical products; on a regular basis the colony receives a wide variety of dosage forms including flavorful, bland, and bitter chewables of varying size and shape. The voluntary full consumption of the sugar placebo was somewhat higher in the mixed breed colony (61.4\% consumption compared to $45 \%$ in the beagle colony). This is not surprising as the laboratory colony is exposed to negative controls (bitter tablets) to maintain selectivity.

For development of a veterinary medication, first choice was assessed in the mixed breed dog colony by completing a preference study. Study 4 was used to document a prehension preference between two formulations. Since the animal was not allowed to consume both dosage forms in the preference study, an acceptance study was also completed to understand consumption and compliance. In the preference study, the dogs chose the tablets with Aromas 1, 2, or 4 two times more often than the sugar placebo control. While in the acceptance study, 
the consumption of tablets with aroma (66.7\% - 68.3\% full consumption) was similar to the control (61.4\% consumption). This is important to note as a preference study in the veterinary pharmaceutical field identifies first choice or first prehension rather than preference in terms of consumption. Interestingly, the tablet with Aroma 3 had similar acceptance to the control, but was prehended first by dogs less often. The tablets with aroma were equal in total amount of aroma added, but Aroma 3 tablets were comprised of a half-measure of Aroma 2 combined with the same amount of another aroma. Therefore, a minimum amount of each type of aroma may be needed to entice the dogs to prehend the dosage form first.

It is critical for oral veterinary medicinal dosage forms to be palatable [5], as complete ingestion is vital for pet therapies to be effective. Many medication failures can be attributed to poor compliance due to the inconvenience of dosage form (such as poking a pill down the throat or hiding a tablet in a food item). This can be inconvenient for the pet owner and could strain the relationship between pet owner and pet. More stressful situations increase the need for improved dosage forms even further, as when pet owners are challenged with chronic treatments that are not palatable to their pet. This paper demonstrates that acceptance palatability studies are important for enabling pet owners to successfully and easily administer products through voluntary consumption of a dosage form.

\section{Conflicts of Interest}

The authors declare no conflicts of interest regarding the publication of this paper.

\section{References}

[1] Committee for Medicinal Products for Veterinary Use (CVMP) (2014) Guideline on the Demonstration of Palatability of Veterinary Medicinal Products. European Medicines Agency, London, 1-7.

http://www.ema.europa.eu/docs/en_GB/document_library/Scientific_guideline/201 4/07/WC500170030.pdf

[2] Courbet, T., Bour, S., Rochet, J., Thibault-Fayard, A. and Boda, C. (2008) Comparison of the Acceptance of 2 Anthelmintic Formulations, in the Dog. Revue De Médecine Vétérinaire, 10, 508-513. https://eurekamag.com/research/030/646/030646269.php

[3] Zemirline, C., Beranger, J., Gobbi, S. and Cissay, E. (2009) Comparative Palatability of a New Formulation and Two Commercial Formulations of Benazepril in Dogs. Revue de Médecine Vétérinaire, 160, 275-281. https://www.researchgate.net/publication/289186699_Comparative_palatability_of_ a_new_formulation_and_two_commercial_formulations_of_benazepril_in_dogs

[4] Payne-Johnson, M., Maitland, T.P., Bullard, J. and Gossellin, J. (2006) Comparative Palatability of Three Commercial Formulations of Carprofen and One Commercial Formulation of Firocoxib in Dogs. Revue De Médecine Vétérinaire, 157, 431-440. http://cat.inist.fr/?aModele $=$ afficheN\&cpsidt $=18257897$

[5] Thombre, A.G. (2004) Oral Delivery of Medications to Companion Animals Palata- 
bility Considerations. Advanced Drug Delivery Reviews, 56, 1399-413.

https://www.ncbi.nlm.nih.gov/pubmed/15191789

https://doi.org/10.1016/j.addr.2004.02.012

[6] AFB International (2018) Principles of Pet Food Palatability. AFB International, St. Charles. 